International Journal of Pure and Applied Mathematics

Volume 98 No. 3 2015, 365-374

ISSN: 1311-8080 (printed version); ISSN: 1314-3395 (on-line version)

url: http://www.ijpam.eu

doi: http://dx.doi.org/10.12732/ijpam.v98i3.9

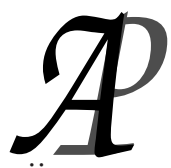

ijpam.eu

\title{
POSITIVE SOLUTIONS FOR NONLINEAR EIGENVALUE PROBLEMS
}

\author{
Hongyan Guan ${ }^{1}$, Yan $\mathrm{Hao}^{2}$ \\ ${ }^{1,2}$ School of Mathematics and Systems Science \\ Shenyang Normal University \\ Shenyang, 110034, P.R. CHINA
}

Abstract: In this paper, by using the Krasnoselskii fixed point theorem, we investigate the existence of positive solutions for the following nonlinear eigenvalue problem:

$$
\left\{\begin{array}{l}
u^{\prime \prime}(t)+a(t) u^{\prime}(t)+b(t) u(t)+\lambda h(t) f(t, u(t))=0, \quad t \in\left[t_{1}, t_{2}\right], \\
u\left(t_{1}\right)=0, \quad \alpha u(\eta)=u\left(t_{2}\right),
\end{array}\right.
$$

where $t_{1}<\eta<t_{2}$ and $0<\alpha \phi_{1}(\eta)<1$ and $\phi_{1}$ is the unique solution of the linear boundary value problem:

$$
\left\{\begin{array}{l}
u^{\prime \prime}(t)+a(t) u^{\prime}(t)+b(t) u(t)=0, \quad t \in\left[t_{1}, t_{2}\right] \\
u\left(t_{1}\right)=0, \quad u\left(t_{2}\right)=1
\end{array}\right.
$$

AMS Subject Classification: $34 \mathrm{~B} 15$

Key Words: positive solution, nonlinear eigenvalue problem, cone, fixed point

\section{Introduction}

In the past few years, there has been much attention focused on the existence of positive solutions for three-point boundary value problems. Il'in and Moiseev [1] studied the existence of solutions for a linear second order differential

Received: October 6, 2014

(C) 2015 Academic Publications, Ltd.

${ }^{\S}$ Correspondence author url: www.acadpubl.eu 
equation. Gupta [2] investigated three-point boundary value problems for nonlinear ordinary differential equations. Ma [3, 4] and Marano [7] established the existence of positive solutions for nonlinear three-point boundary-value problems. Recently, Ma and Wang [5] used the Krasnoselskii fixed point theorem to prove the existence of at least one positive solution for the following nonlinear three-point boundary-value problem:

$$
\left\{\begin{array}{l}
u^{\prime \prime}(t)+a(t) u^{\prime}(t)+b(t) u(t)+h(t) f(u)=0, t \in(0,1), \\
u(0)=0, \alpha u(\eta)=u(1),
\end{array}\right.
$$

where $0<\eta<1, h \in C([0,1],[0, \infty))$ satisfies that there exists $x_{0} \in[0,1]$ such that $h\left(x_{0}\right)>0$ and $f \in C([0,1],[0, \infty)), 0<\alpha \phi_{1}(\eta)<1$ and $\phi_{1}$ is the unique solution of the linear boundary value problem:

$$
\left\{\begin{array}{l}
u^{\prime \prime}(t)+a(t) u^{\prime}(t)+b(t) u(t)=0, t \in(0,1), \\
u(0)=0, u(1)=1 .
\end{array}\right.
$$

Motivated and inspired by the results in [2]-[5] and [7], in this paper, by using the Krasnoselskii fixed point theorem, we study the existence of positive solutions for the following nonlinear eigenvalue problem:

$$
\left\{\begin{array}{l}
u^{\prime \prime}(t)+a(t) u^{\prime}(t)+b(t) u(t)+\lambda h(t) f(t, u(t))=0, t \in\left[t_{1}, t_{2}\right] \\
u\left(t_{1}\right)=0, \alpha u(\eta)=u\left(t_{2}\right)
\end{array}\right.
$$

where $t_{1}<\eta<t_{2}, 0<\alpha \phi_{1}(\eta)<1$ and $\phi_{1}$ is the unique solution of the linear boundary value problem:

$$
\left\{\begin{array}{l}
u^{\prime \prime}(t)+a(t) u^{\prime}(t)+b(t) u(t)=0, t \in\left[t_{1}, t_{2}\right] \\
u\left(t_{1}\right)=0, u\left(t_{2}\right)=1
\end{array}\right.
$$

and $a, b, f$ and $h$ satisfy the following conditions:

$\left(H_{1}\right) f \in C\left(\left[t_{1}, t_{2}\right] \times[0,+\infty),[0,+\infty)\right)$;

$\left(H_{2}\right) h \in C\left(\left[t_{1}, t_{2}\right],[0,+\infty)\right)$ and there exists $x_{0} \in\left[t_{1}, t_{2}\right]$ such that $h\left(x_{0}\right)>$ 0 ;

$\left(H_{3}\right) a \in C\left[t_{1}, t_{2}\right], b \in C\left(\left[t_{1}, t_{2}\right],(-\infty, 0)\right)$.

\section{Preliminaries}

In order to prove our main results, we need the following lemmas. 
Lemma 1. ([6]) Let $E$ is a Banach space and let $K \subset E$ be a cone. Assume that $\Omega_{1}, \Omega_{2}$ are open bounded subsets of $E$ with $0 \in \Omega_{1}, \bar{\Omega}_{1} \subset \Omega_{2}$, and $A: K \cap\left(\bar{\Omega}_{2} \backslash \Omega_{1}\right) \rightarrow K$ is a completely continuous operator such that

(a) $\|A u\| \leq\|u\|, u \in K \cap \partial \bar{\Omega}_{1}$ and $\|A u\| \geq\|u\|, u \in K \cap \partial \bar{\Omega}_{2}$; or

(b) $\|A u\| \geq\|u\|, u \in K \cap \partial \bar{\Omega}_{1}$ and $\|A u\| \leq\|u\|, u \in K \cap \partial \bar{\Omega}_{2}$.

Then $A$ has at least one fixed point in $K \cap\left(\bar{\Omega}_{2} \backslash \Omega_{1}\right)$.

The arguments of Lemmas 2-6 below are similar to that of the correspondent results in [5], we omit them here.

Lemma 2. Assume that $\left(H_{3}\right)$ hold. Let $\phi_{1}$ and $\phi_{2}$ be the solutions of

$$
\left\{\begin{array}{l}
u^{\prime \prime}(t)+a(t) u^{\prime}(t)+b(t) u(t)=0, t \in\left[t_{1}, t_{2}\right] \\
u\left(t_{1}\right)=0, u\left(t_{2}\right)=1
\end{array}\right.
$$

and

$$
\left\{\begin{array}{l}
u^{\prime \prime}(t)+a(t) u^{\prime}(t)+b(t) u(t)=0, t \in\left[t_{1}, t_{2}\right], \\
u\left(t_{1}\right)=1, u\left(t_{2}\right)=0
\end{array}\right.
$$

respectively. Then

(a) $\phi_{1}$ is strictly increasing on $\left[t_{1}, t_{2}\right]$;

(b) $\phi_{2}$ is strictly decreasing on $\left[t_{1}, t_{2}\right]$.

Lemma 3. Assume that $\left(H_{3}\right)$ hold. Then (2) and (3) have a unique solution, respectively.

$\left(H_{4}\right) 0<\alpha \phi_{1}(\eta)<1$.

Lemma 4. Let $\left(H_{3}\right),\left(H_{4}\right)$ hold and $y \in C\left[t_{1}, t_{2}\right]$. Then the problem

$$
\left\{\begin{array}{l}
u^{\prime \prime}(t)+a(t) u^{\prime}(t)+b(t) u(t)+y(t)=0, t \in\left[t_{1}, t_{2}\right], \\
u\left(t_{1}\right)=0, \alpha u(\eta)=u\left(t_{2}\right),
\end{array}\right.
$$

is equivalent to the integral equation

$$
u(t)=\int_{t_{1}}^{t_{2}} G(t, s) p(s) y(s) d s+A \phi_{1}(t)
$$

where

$$
A=\frac{\alpha}{1-\alpha \phi_{1}(\eta)} \int_{t_{1}}^{t_{2}} G(\eta, s) p(s) y(s) d s, \quad p(t)=\exp \left(\int_{t_{1}}^{t} a(s) d s\right),
$$




$$
\rho=\phi_{1}^{\prime}\left(t_{1}\right), \quad G(t, s)=\frac{1}{\rho}\left\{\begin{array}{l}
\phi_{1}(t) \phi_{2}(s), s>t, \\
\phi_{1}(s) \phi_{2}(t), s \leq t .
\end{array}\right.
$$

Moreover, $u(t) \geq 0$ for $t \in\left[t_{1}, t_{2}\right]$ provided that $y(t) \geq 0$.

Lemma 5. Let $\left(H_{3}\right),\left(H_{4}\right)$ hold, $y \in C\left[t_{1}, t_{2}\right]$ and $y$ be nonnegative. Then the unique solution of (4) satisfies that

$$
u(t) \geq\|u\| q(t), \quad t \in\left[t_{1}, t_{2}\right],
$$

where $q(t)=\min \left\{\frac{\phi_{1}(t)}{\left\|\phi_{1}\right\|}, \frac{\phi_{2}(t)}{\left\|\phi_{2}\right\|}\right\}, t \in\left[t_{1}, t_{2}\right]$.

Lemma 6. Assume that $\left(H_{3}\right),\left(H_{4}\right)$ hold. Let $y \in C\left[t_{1}, t_{2}\right]$ and be nonnegative. Then for any constants $l_{1}$ and $l_{2}$ with $t_{1}<l_{1}<l_{2}<t_{2}$, there exists $\gamma=\min _{t \in\left[l_{1}, l_{2}\right]} q(t)$ such that the unique solution of the problem (4) satisfies that

$$
u(t) \geq \gamma\|u\|, \quad t \in\left[l_{1}, l_{2}\right],
$$

where $q(t)$ is as in Lemma 5.

\section{Main Results}

In this section, we will use Lemma 1 to prove the existence of positive solutions for the nonlinear eigenvalue problem (1). For fixed $l_{1}, l_{2}$ such that $t_{1}<l_{1}<$ $l_{2}<t_{2}$, set

$$
\begin{aligned}
& \underline{f}_{0}=\liminf _{u \rightarrow 0^{+}} \frac{1}{u} \min \left\{f(t, u): t \in\left[l_{1}, l_{2}\right]\right\}, \\
& \bar{f}_{0}=\limsup _{u \rightarrow 0^{+}} \frac{1}{u} \max \left\{f(t, u): t \in\left[t_{1}, t_{2}\right]\right\}, \\
& \underline{f}_{\infty}=\liminf _{u \rightarrow \infty} \frac{1}{u} \min \left\{f(t, u): t \in\left[l_{1}, l_{2}\right]\right\}, \\
& \bar{f}_{\infty}=\limsup _{u \rightarrow \infty} \frac{1}{u} \max \left\{f(t, u): t \in\left[t_{1}, t_{2}\right]\right\} .
\end{aligned}
$$

Our main results are as follows:

Theorem 7. Let $\left(H_{1}\right)-\left(H_{4}\right)$ hold. Then for each $\lambda$ satisfying

$$
\frac{1}{\underline{f}_{0} \gamma \int_{l_{1}}^{l_{2}} G\left(x_{0}, s\right) p(s) h(s) d s}<\lambda<\frac{1}{\bar{f}_{\infty} B},
$$


where $B=\int_{t_{1}}^{t_{2}} G(s, s) p(s) h(s) d s+\frac{\alpha}{1-\alpha \phi_{1}(\eta)} \int_{t_{1}}^{t_{2}} G(\eta, s) p(s) h(s) d s$ and $\gamma$ is defined in Lemma 6, the nonlinear eigenvalue problem (1) has at least one positive solution.

Proof. It follows from Lemma 4 that the nonlinear eigenvalue problem (1) has a solution $u=u(t)$ if and only if $u$ solves the operator equation

$$
\begin{aligned}
T u(t)= & \lambda\left[\int_{t_{1}}^{t_{2}} G(t, s) p(s) h(s) f(s, u(s)) d s\right. \\
& \left.+\frac{\alpha \phi_{1}(t)}{1-\alpha \phi_{1}(\eta)} \int_{t_{1}}^{t_{2}} G(\eta, s) p(s) h(s) f(s, u(s)) d s\right], t \in\left[t_{1}, t_{2}\right],
\end{aligned}
$$

where $p, G$ and $\rho$ are defined as in Lemma 4. Put

$$
K=\left\{u: u \in C\left[t_{1}, t_{2}\right], u \geq 0, \min _{t \in\left[l_{1}, l_{2}\right]} u(t) \geq \gamma\|u\|\right\} .
$$

It is clear that $K$ is a cone in $C\left[t_{1}, t_{2}\right]$. Moreover, by Lemma 3 and Lemma 5 , we get that $T K \subseteq K$. It is also easy to prove that $T: K \rightarrow K$ is completely continuous.

Let $\lambda$ be given as in (5) and choose $\varepsilon>0$ such that

$$
\frac{1}{\left(\underline{f}_{0}-\varepsilon\right) \gamma \int_{l_{1}}^{l_{2}} G\left(x_{0}, s\right) p(s) h(s) d s} \leq \lambda \leq \frac{1}{\left(\bar{f}_{\infty}+\varepsilon\right) B} .
$$

It follows from $\underline{f}_{0}=\liminf _{u \rightarrow 0^{+}} \frac{1}{u} \min \left\{f(t, u): t \in\left[l_{1}, l_{2}\right]\right\}$ that there exists an $H_{1}>0$ such that $f(t, u) \geq\left(\underline{f}_{0}-\varepsilon\right) u$ for $0<u \leq H_{1}$ and $t \in\left[l_{1}, l_{2}\right]$. Pick $u \in K$ with $\|u\|=H_{1}$. Using (6) and (7), we have

$$
\begin{aligned}
T u\left(x_{0}\right) & \geq \lambda \int_{t_{1}}^{t_{2}} G\left(x_{0}, s\right) p(s) h(s) f(s, u(s)) d s \\
& \geq \lambda \int_{l_{1}}^{l_{2}} G\left(x_{0}, s\right) p(s) h(s) f(s, u(s)) d s \\
& \geq \lambda\left(\underline{f}_{0}-\varepsilon\right) \gamma\|u\| \int_{l_{1}}^{l_{2}} G\left(x_{0}, s\right) p(s) h(s) d s \geq\|u\| .
\end{aligned}
$$

Therefore, $\|T u\| \geq\|u\|$ for $u \in K \cap \partial \Omega_{1}$, where $\Omega_{1}=\left\{x \in C\left[t_{1}, t_{2}\right]:\|x\|<H_{1}\right\}$.

In view of $\bar{f}_{\infty}=\limsup _{u \rightarrow \infty} \frac{1}{u} \max \left\{f(t, u): t \in\left[t_{1}, t_{2}\right]\right\}$, we derive that there exists an $\bar{H}_{2}>H_{1}$ such that $f(t, u) \leq\left(f_{\infty}+\varepsilon\right) u$ for $u \geq \bar{H}_{2}$ and $t \in\left[t_{1}, t_{2}\right]$. We consider the following cases: 
Case 1. Suppose that $f$ is bounded. It is clear that there exists $M>0$ with $f(t, u) \leq M$ for all $u \in[0,+\infty), t \in\left[t_{1}, t_{2}\right]$. Let $H_{2}=\max \left\{2 \bar{H}_{2}, \lambda M B\right\}$. Take $u \in K$ with $\|u\|=H_{2}$. Since $G(t, s) \leq G(s, s)$ for $t, s \in\left[t_{1}, t_{2}\right]$, we infer that

$$
\begin{aligned}
T u(t) \leq & \lambda\left[\int_{t_{1}}^{t_{2}} G(s, s) p(s) h(s) f(s, u(s)) d s\right. \\
& \left.+\frac{\alpha \phi_{1}(t)}{1-\alpha \phi_{1}(\eta)} \int_{t_{1}}^{t_{2}} G(\eta, s) p(s) h(s) f(s, u(s)) d s\right] \\
\leq & \lambda M B, \quad t \in\left[t_{1}, t_{2}\right] .
\end{aligned}
$$

As a result, $\|T u\| \leq\|u\|$ for $u \in K \cap \partial \Omega_{2}$, where $\Omega_{2}=\left\{x \in C\left[t_{1}, t_{2}\right]:\|x\|<H_{2}\right\}$.

Case 2. Suppose that $f$ is unbounded. It follows that there exist $H_{2} \geq$ $\max \left\{\bar{H}_{2}, 2 H_{1}\right\}$ and $t_{0} \in\left[t_{1}, t_{2}\right]$ such that $f(t, u) \leq f\left(t_{0}, H_{2}\right)$ for $0 \leq u \leq H_{2}$ and $t \in\left[t_{1}, t_{2}\right]$. Choose $u \in K$ with $\|u\|=H_{2}$, by (7), we have

$$
\begin{aligned}
T u(t) \leq & \lambda\left[\int_{t_{1}}^{t_{2}} G(s, s) p(s) h(s) f(s, u(s)) d s\right. \\
& \left.+\frac{\alpha}{1-\alpha \phi_{1}(\eta)} \int_{t_{1}}^{t_{2}} G(\eta, s) p(s) h(s) f(s, u(s)) d s\right] \\
\leq & \lambda f\left(t_{0}, H_{2}\right) B \leq \lambda\left(\bar{f}_{\infty}+\varepsilon\right) H_{2} B \leq H_{2}=\|u\|, \quad t \in\left[t_{1}, t_{2}\right] .
\end{aligned}
$$

That is, $\|T u\| \leq\|u\|$ for all $u \in K \cap \partial \Omega_{2}$, where $\Omega_{2}=\left\{x \in C\left[t_{1}, t_{2}\right]:\|x\|<H_{2}\right\}$.

Consequently, Lemma 1 guarantees that the nonlinear eigenvalue problem (1) has at least one positive solution in $K$. This completes the proof.

Theorem 8. Assume that $\left(H_{1}\right)-\left(H_{4}\right)$ hold. Let $B$ and $\gamma$ be as in Theorem 7. Then for each $\lambda$ satisfying

$$
\frac{1}{\underline{f}_{\infty} \gamma \int_{l_{1}}^{l_{2}} G\left(x_{0}, s\right) p(s) h(s) d s}<\lambda<\frac{1}{\bar{f}_{0} B},
$$

the nonlinear eigenvalue problem (1) has at least one positive solution.

Proof. Let $K$ and $T$ be as in the proof of Theorem 7. Let $\lambda$ be given as in (8) and choose $\varepsilon>0$ satisfying

$$
\frac{1}{\left(\underline{f}_{\infty}-\varepsilon\right) \gamma \int_{l_{1}}^{l_{2}} G\left(x_{0}, s\right) p(s) h(s) d s} \leq \lambda \leq \frac{1}{\left(\bar{f}_{0}+\varepsilon\right) B} .
$$


Considering $\bar{f}_{0}$, there exists an $H_{1}>0$ such that $f(t, u) \leq\left(\bar{f}_{0}+\varepsilon\right) u$ for $0<$ $u \leq H_{1}, t \in\left[t_{1}, t_{2}\right]$. Pick $u \in K$ with $\|u\|=H_{1}$. In view of (9), we have

$$
\begin{aligned}
T u(t) \leq & \lambda\left[\int_{t_{1}}^{t_{2}} G(s, s) p(s) h(s) f(s, u(s)) d s\right. \\
& \left.+\frac{\alpha}{1-\alpha \phi_{1}(\eta)} \int_{t_{1}}^{t_{2}} G(\eta, s) p(s) h(s) f(s, u(s)) d s\right] \\
\leq & \lambda\left(\bar{f}_{0}+\varepsilon\right) B\|u\| \leq\|u\|, \quad t \in\left[t_{1}, t_{2}\right] .
\end{aligned}
$$

Consequently, $\|T u\| \leq\|u\|$ for $u \in K \cap \partial \Omega_{1}$, where $\Omega_{1}=\left\{x \in C\left[t_{1}, t_{2}\right]:\|x\|<\right.$ $\left.H_{1}\right\}$.

Note that $\underline{f}_{\infty}=\liminf _{u \rightarrow \infty} \frac{1}{u} \min \left\{f(t, u): t \in\left[l_{1}, l_{2}\right]\right\}$ ensures that there exists an $\bar{H}_{2}>0$ satisfying $f(t, u) \geq\left(\underline{f}_{\infty}-\varepsilon\right) u$ for all $u \geq \bar{H}_{2}$ and $t \in\left[l_{1}, l_{2}\right]$. Let $H_{2}=\max \left\{2 H_{1}, \gamma^{-1} \bar{H}_{2}\right\}$ and $\Omega_{2}=\left\{x \in C\left[t_{1}, t_{2}\right]:\|x\|<H_{2}\right\}$. For $u \in K$ with $\|u\|=H_{2}$, it follows from $\min _{l_{1} \leq t \leq l_{2}} u(t) \geq \bar{H}_{2}$ that

$$
\begin{aligned}
T u\left(x_{0}\right) & \geq \lambda \int_{t_{1}}^{t_{2}} G\left(x_{0}, s\right) p(s) h(s) f(s, u(s)) d s \\
& \geq \lambda \int_{l_{1}}^{l_{2}} G\left(x_{0}, s\right) p(s) h(s) f(s, u(s)) d s \\
& \geq \lambda\left(\underline{f}_{\infty}-\varepsilon\right) \gamma\|u\| \int_{l_{1}}^{l_{2}} G\left(x_{0}, s\right) p(s) h(s) d s \geq\|u\| .
\end{aligned}
$$

This is, $\|T u\| \geq\|u\|$ for $u \in K \cap \partial \Omega_{2}$. Lemma 1 ensures that $T$ has a fixed point $u(t) \in K \cap\left(\bar{\Omega}_{2} \backslash \Omega_{1}\right)$. Hence the nonlinear eigenvalue problem (1) possesses at least one positive solution in $K$. This completes the proof.

Theorem 9. Let $\left(H_{1}\right)-\left(H_{4}\right)$ hold, and $B$ and $\gamma$ be as in Theorem 7 . Assume that there exist positive constants $p_{1}, p_{2}, q_{1}$ and $q_{2}$ with $p_{1}<q_{1}<$ $q_{2}<p_{2}$ satisfying

$$
f(t, u) \leq \bar{f}_{\infty} p_{1}, \quad(t, u) \in\left[t_{1}, t_{2}\right] \times\left[0, q_{1}\right],
$$

and

$$
f(t, u) \geq \underline{f}_{0} p_{2}, \quad(t, u) \in\left[l_{1}, l_{2}\right] \times\left[q_{2},+\infty\right) .
$$

Then for each $\lambda$ satisfying (5), the nonlinear eigenvalue problem (1) has at least two positive solutions. 
Proof. Let $K$ and $T$ be as in the proof of Theorem 7. Set $P_{1}=\{x \in$ $\left.C\left[t_{1}, t_{2}\right]:\|x\|<p_{1}\right\}, P_{2}=\left\{x \in C\left[t_{1}, t_{2}\right]:\|x\|<p_{2}\right\}$ and take $u \in K$ with $\|u\|=p_{1}$. It follows from (6) and (10) that

$$
\begin{aligned}
T u(t) \leq & \lambda\left[\int_{t_{1}}^{t_{2}} G(s, s) p(s) h(s) f(s, u(s)) d s\right. \\
& \left.+\frac{\alpha}{1-\alpha \phi_{1}(\eta)} \int_{t_{1}}^{t_{2}} G(\eta, s) p(s) h(s) f(s, u(s)) d s\right] \\
\leq & \lambda p_{1} \bar{f}_{\infty} B \leq p_{1} .
\end{aligned}
$$

That is, $\|T u\| \leq\|u\|$ for $u \in K \cap \partial P_{1}$.

By $\underline{f}_{0}=\liminf \operatorname{in}_{u \rightarrow 0^{+}} \frac{1}{u} \min \left\{f(t, u): t \in\left[l_{1}, l_{2}\right]\right\}$, we infer that there exists an $H_{1}$ such that $0<H_{1}<\frac{1}{2} p_{1}$ and $f(t, u) \geq\left(\underline{f}_{0}-\varepsilon\right) u$ for $0<u \leq H_{1}, t \in\left[l_{1}, l_{2}\right]$. As in the proof of Theorem 7, we get that $\|T u\| \geq\|u\|$ for all $u \in K \cap \partial \Omega_{1}$, where $\Omega_{1}=\left\{x \in C\left[t_{1}, t_{2}\right]:\|x\|<H_{1}\right\}$. It follows from Lemma 1 that $T$ has a fixed point $u_{1} \in \bar{P}_{1} \backslash \Omega_{1}$ with $H_{1} \leq\left\|u_{1}\right\| \leq p_{1}$.

Now choose $u \in K$ with $\|u\|=p_{2}$. In view of (6) and (11), we get that

$$
T u\left(x_{0}\right) \geq \lambda \int_{t_{1}}^{t_{2}} G\left(x_{0}, s\right) p(s) h(s) f(s, u(s)) d s \geq p_{2} .
$$

Therefore, $\|T u\| \geq\|u\|$ for $u \in K \cap \partial P_{2}$.

Since $\bar{f}_{\infty}=\limsup _{u \rightarrow \infty} \frac{1}{u} \max \left\{f(t, u): t \in\left[t_{1}, t_{2}\right]\right\}$, it follows that there exists $\bar{H}_{2}>2 p_{2}$ such that $f(t, u) \leq\left(\bar{f}_{\infty}+\varepsilon\right) u$ for $u \geq \bar{H}_{2}$ and $t \in\left[t_{1}, t_{2}\right]$. As in the proof of Theorem 7, we derive that $\|T u\| \leq\|u\|$ for $u \in K \cap \partial \Omega_{2}$, where $\Omega_{2}=\left\{x \in C\left[t_{1}, t_{2}\right]:\|x\|<H_{2}\right\}$ and $H_{2}>\bar{H}_{2}$. Lemma 1 ensures that $T$ has a fixed point $u_{2} \in \bar{\Omega}_{2} \backslash P_{2}$ with $p_{2} \leq\left\|u_{2}\right\| \leq H_{2}$. As a result, $T$ has two different fixed points $u_{1}, u_{2}$ in $K$. That is, the nonlinear eigenvalue problem (1) has at least two positive solutions $u_{1}$ and $u_{2}$. This completes the proof.

Similarly, we obtain the following result:

Theorem 10. Let $\left(H_{1}\right)-\left(H_{4}\right)$ hold, and $B$ and $\gamma$ be as in Theorem 7 . Assume that there exist positive constants $p_{1}, p_{2}, q_{1}$ and $q_{2}$ with $p_{1}<q_{1}<q_{2}<$ $p_{2}$ satisfying

$$
f(t, u) \geq \underline{f}_{\infty} p_{1}, \quad(t, u) \in\left[l_{1}, l_{2}\right] \times\left[0, q_{1}\right]
$$

and

$$
f(t, u) \leq \bar{f}_{0} p_{2}, \quad(t, u) \in\left[t_{1}, t_{2}\right] \times\left[q_{2},+\infty\right) .
$$

Then for each $\lambda$ satisfying (8), the nonlinear eigenvalue problem (1) has at least two positive solutions. 


\section{An Example}

In this section, we construct an example to explain Theorem 7 .

Example 11. Consider the nonlinear eigenvalue problem

$$
\left\{\begin{array}{l}
u^{\prime \prime}(t)+\frac{1}{t} u^{\prime}(t)-\frac{1}{t^{2}} u(t)+\lambda \frac{576 t^{2}\left(u^{2}(t)+180 u(t)\right)}{u(t)+2}=0, t \in\left[\frac{1}{5}, 1\right], \\
u\left(\frac{1}{5}\right)=0, \quad u\left(\frac{2}{3}\right)=2 u(1) .
\end{array}\right.
$$

It is easy to verify that $\phi_{1}(t)=\frac{25}{24} t-\frac{1}{24 t}, \phi_{2}(t)=-\frac{5}{24} t+\frac{5}{24 t}$ and $1-\frac{1}{2} \phi_{1}\left(\frac{2}{3}\right)=\frac{197}{288}$. Take $l_{1}=\frac{1}{3}, l_{2}=\frac{3}{4}, x_{0}=\frac{2}{3}$ and $\gamma=\frac{35}{288}$. Let

$$
f(t, u)=\frac{\left(u^{2}+180 u\right) t}{u+2},(t, u) \in\left[\frac{1}{5}, 1\right] \times[0,+\infty), h(t)=576 t, t \in\left[\frac{1}{5}, 1\right] .
$$

It is obvious that $f \in C\left(\left[\frac{1}{5}, 1\right] \times[0,+\infty),[0,+\infty)\right)$ and

$$
\begin{aligned}
& \underline{f}_{0}=\liminf _{u \rightarrow 0^{+}} \frac{1}{u} \min \left\{\frac{\left(u^{2}+180 u\right) t}{u+2}: t \in\left[\frac{1}{3}, \frac{3}{4}\right]\right\}=30, \\
& \bar{f}_{\infty}=\limsup _{u \rightarrow \infty} \frac{1}{u} \max \left\{\frac{\left(u^{2}+180 u\right) t}{u+2}: t \in\left[\frac{1}{5}, 1\right]\right\}=1 .
\end{aligned}
$$

If $\frac{1}{50}<\lambda<\frac{1}{15}$, then by Theorem 7, the nonlinear eigenvalue problem (12) possesses at least one positive solution.

\section{References}

[1] V. A. Il'in, E. I. Moiseev, Nonlocal boundary value problem of the second kind for a Sturm-Liouville operator, Differential Equations, 23, No. 8 (1987), 979-987.

[2] C. P. Gupta, Solvability of a three-point nonlinear boundary value problem for a second order ordinary differential equations, J. Math. Anal. Appl., 168, No. 2 (1992), 540-551, doi:10.1016/0022-247X(92)90179-H.

[3] R. Ma, Existence theorems for a second order three-point boundary value problem, J. Math. Anal. Appl., 212, No. 2 (1997), 430-442, doi:10.1006/jmaa.1997.5515. 
[4] R. Ma, Positive solutions of a nonlinear three-point boundary value problem, Electronic Journal of Differential Equations, 1998, No. 34 (1998), 1-8.

[5] R. Ma, H. Wang, Positive solutions of nonlinear three-point boundary value problems, J. Math. Anal. Appl., 279, No. 1 (2003), 216-227, doi:10.1016/S0022-247X(02)00661-3.

[6] M. A. Krasnoselskii, Positive Solutions of Operator Equation, Noordhoff, Groningen (1964).

[7] S. A. Marano, A remark on a second order three-point boundary value problem, J. Math. Anal. Appl., 183, No. 3 (1994), 518-522, doi:10.1006/jmaa.1994.1158. 